\title{
Computerized cognitive testing in patients with type I Gaucher disease: Effects of enzyme replacement and substrate reduction
}

Deborah Elstein, $P h D^{1}$, Judith Guedalia, $P h D^{2}$, Glen M. Doniger, $P h D^{3}$, Ely S. Simon, $M D^{3}$, Vered Antebi, $M A^{2}$, Yael Arnon, $M A^{2}$, and Ari Zimran, $M D^{1}$

\begin{abstract}
Purpose: Because of concern for drug-induced cognitive dysfunction during clinical trials using substrate reduction therapy (miglustat) in type 1 Gaucher disease and because it has been suggested that some patients with type 1 Gaucher disease may develop neurocognitive impairment as part of the natural history, two different batteries of neuropsychological tests were devised to examine these issues. Using these tests, cognitive function was assessed in patients treated with miglustat, in patients receiving enzyme replacement (standard care for symptomatic patients), and in untreated (milder) patients. Methods: For this study, 55/60 patients exposed to miglustat in Israel participated in psychologist-administered testing; 36/55 participated in computerized testing. Of these, 31 enzyme-treated patients and 22 untreated patients participated in the psychologist-administered testing, and 15 enzyme-treated patients and 18 untreated patients participated in computerized testing. The psychologistadministered battery consisted of 18 standard neuropsychological subtests specific to executive and visuospatial functioning. The computerized battery (Mindstreams ${ }^{\circledR}$, NeuroTrax Corp., New York, NY) consisted of 10 subtests tapping multiple cognitive domains. Between-group analyses for each modality compared cognitive performance. Results: In the psychologist-administered testing, patients exposed to miglustat performed significantly less well than the other groups in $5 / 18$ subtests. On the computerized tests, all patients performed comparably to normal controls. Scores in patients exposed to miglustat were higher than in untreated patients, particularly in visuospatial function, whereas enzyme-treated patients performed less well. However, with the exception of visuospatial function, these results were not statistically significant. Conclusions: It is unclear why different testing methods yielded discordant results. Any dysfunction suggested by the current study is apparently subtle and of doubtful clinical relevance given that cognitive status did not interfere with patients' daily intellectual function. The computerized battery has methodological advantages (e.g., language options, objectivity, brevity, and ease of use) that make it well-suited for longitudinal studies, for long-term surveillance of substrate reduction therapy as well as for comparisons with other lysosomal storage disorders and other chronic diseases. These preliminary findings should allay fears of cognitive dysfunction due to short-term miglustat therapy. Genet Med 2005:7(2):124-130.
\end{abstract}

Key Words: Gaucher disease, computerized cognitive testing, enzyme replacement therapy, neuropsychology, substrate reduction therapy, visuospatial defect.

Gaucher disease, the most common sphingolipid storage disorder, was the first lysosomal storage disease to be effectively treated with enzyme replacement therapy (ERT). ${ }^{1}$ Both placenta-derived (alglucerase) and recombinant (imiglucerase) glucocerebrosidase (Genzyme Therapeutics Inc, Cambridge, MA) reduce hepatosplenomegaly, improve hematolog-

From the ${ }^{I}$ Gaucher Clinic and ${ }^{2}$ Neuropsychology Unit, Shaare Zedek Medical Center, Jerusalem, Israel; and the ${ }^{3}$ Department of Clinical Science, NeuroTrax Corporation, New York, NY. Deborah Elstein, PhD, Gaucher Clinic, Shaare Zedek Medical Center, PO.B. 3235, Jerusalem 91031 Israel.

Received: July 13, 2004.

Accepted: November 11, 2004.

DOI: 10.1097/01.GIM.0000153666.23707.BA ical and biochemical parameters of the disease, and ameliorate skeletal disease and other visceral features among patients with type 1 Gaucher disease, the nonneuronopathic form. ${ }^{2}$ In Israel, only patients who meet Ministry of Health criteria for severity of disease are approved for treatment. In 1996, Radin suggested that this "... mode of treatment should be replaceable with a suitable enzyme inhibitor that simply slows formation of the lipid, and matches the rate of synthesis with the rate of the defective, slowly working beta-glucosidase."3 In April 2000, the results of the first clinical trial with an oral substrate inhibitor, the iminosugar $\mathrm{N}$-butyldeoxynojirimycin (miglustat; $\mathrm{Ox}$ ford GlycoSciences, Oxford, UK), as monotherapy in type 1 Gaucher disease were reported. ${ }^{4}$ Therapy was effective primarily in reducing organomegaly and elevated levels of serum chi- 
totriosidase and was tolerated in most patients with manageable side effects, particularly diarrhea $(85 \%)$ and weight loss (65\%). Further trials of miglustat were initiated to assess lowdose safety and efficacy ${ }^{5}$ as well as its use in maintenance of patients who had heretofore received ERT. ${ }^{6}$ Between 1998 to 2002, 90 patients with Gaucher disease in six medical centers were treated with miglustat. In these studies, generally reversible neurotoxicity was observed in some patients: tremor (30\%), peripheral neuropathy $(15 \%-20 \%) .7,8$

In April 2002, after 34 months of treatment with no major side effects, a 67-year old Israeli polylinguist participating in the original miglustat trial complained of inability to learn a new language, forgetfulness, and lack of characteristic organizational abilities. Because a drug-induced memory loss could not be excluded, the local Ethics Committee suspended miglustat treatment in Israel pending further evaluation of these putative cognitive symptoms. A battery of tests related to frontal and parietal lobe dysfunction, the areas affected in the above patient, was devised and administered during May-August 2002 with a conclusion that there was no correlation between length of exposure to miglustat and decreased cognitive function and that the cognitive complaints were probably not miglustat-related. Consequently, the clinical trial of miglustat in Israel was reinstated in November 2002.

Miglustat (Zavesca, Actelion Pharmaceuticals Inc., Allschwil, Switzerland and Teva Pharmaceuticals Industries Ltd., Netanya, Israel) is now licensed in Europe and Israel for symptomatic patients with mild to moderate type 1 Gaucher disease who are unsuitable for ERT $^{9}$ and in the United States for symptomatic patients with mild to moderate disease in whom ERT is not a therapeutic option. ${ }^{10}$

As a condition for licensure, postapproval long-term surveillance of cognitive function, specifically visuospatial and executive functioning, in patients receiving Zavesca was required by the various regulatory agencies. However, even before this requirement, a study of traditional neuropsychological testing was initiated in all Israeli patients with type 1 Gaucher disease treated with miglustat compared to age- and sex-matched patients receiving ERT and patients who had never been treated with either drug. Because of concern that test results were unduly influenced by methodological idiosyncrasies (e.g., single language [Hebrew] administration and the need for translators for other native-language speakers; time of administration exceeding more than 3 hours on average; the use of non-Israeli norms for some subtests), most patients were also tested with a self-administered, standardized computerized battery with language options and custom-normalized to a mainly Israeli group. In this article, we report results for both methodologies and discuss differences in outcomes and the clinical significance of our findings.

\section{MATERIALS AND METHODS}

\section{Traditional neuropsychological battery}

Starting in May 2002, all 60 patients who had participated in the original miglustat trial in Israel were encouraged to partic- ipate in a psychologist-administered study; the only patients who were not included were two non-Israeli nationals and three patients who were out of the country during the testing period. Additional adult patients with type 1 Gaucher disease who had either been receiving ERT or no treatment were asked to participate; this cohort was culled from those patients who appeared for routine evaluations during this 6-month period, albeit some bias may have occurred since patients who declined to volunteer were not asked for an explanation. No records tracked patients who did not agree to participate. No patient in any of the three groups had a history of cognitive impairment. All testing was completed by November 2002.

Ethics (Helsinki) Committee approval was mandated for this study in order to reinstate the clinical trial.

The following tests were administered in the standard way to each patient individually: for general cognitive intellectual abilities, parts of the Wechsler Adult Intelligence Scale Revised $^{11}$ and the Mini Mental State Examination ${ }^{12}$; for language function, the Word Fluency (FAS) and Semantic Fluency (Categories, Animals) Tests ${ }^{13}$; for visuospatial/visuomotor/visuoconstructional abilities and nonverbal memory ability, the Rey Osterreith Complex Figure Test and Recall ${ }^{14}$ and Trail Making Tests A and B (TMTA, TMTB) ${ }^{15}$; for verbal learning ability and verbal memory, the Rey Auditory Verbal Learning Test (RAVLT1-6) including long-term Recall and Recognition (RAVLT7 and RAVLT8) ${ }^{16}$; for executive functioning, the Tower of $\mathrm{Hanoi}^{17}$; and for higher cognitive functioning, the Wisconsin Card Sorting Test, ${ }^{18}$ making a total of 18 subtests

All tests were individually administered in the native language of the patient (Hebrew, Russian, or English) by psychologists (V.A. and Y.A.) with the assistance of translators when necessary. Psychologists and translators were blinded as to the status of the patient.

\section{Statistical analysis for neuropsychological battery}

Means, standard deviations (SD), and z-scores were calculated (and compared to the $\mathrm{z}$-scores of standard normograms). One-way analysis of variance (ANOVA) using a 95\% confidence interval was used to assess between-group differences. Pearson chi-square test (with continuity correction) was computed to correlate performance with length of exposure to miglustat. Because the entire cohort performed one to two standard deviations below the standardized norms, -1.5 and -2.0 $\mathrm{z}$-scores were separately examined as cut-off points for classification as cognitively impaired.

\section{Mindstreams}

All patients with type 1 Gaucher disease appearing at the Gaucher Clinic for routine evaluations after November 2002 were asked to participate in this phase of the study by completing the Mindstreams ${ }^{\circledR}$ (NeuroTrax Corp., NY) computerized cognitive battery for detection of mild impairment (i.e., the Global Assessment Battery ${ }^{19}$ ). Performance in patients was compared with that of individuals similar in age, years of education, and gender to the patients with Gaucher disease in the 
present study who were drawn from a database of Mindstreams data collected in controlled research studies. ${ }^{19,20}$

Performance in patients who had never been treated was compared with that of patients receiving ERT and with those on miglustat. Tests were available in English, Hebrew, and Russian and were administered in the patients' primary language (i.e., language most comfortable speaking or language used most often). Ethics Committee approval was obtained for the study, and informed consent was obtained from all participants.

Mindstreams data were uploaded to the NeuroTrax central server, where data processing occurred, during which aggregate outcome parameters were computed from raw single-trial data. ${ }^{19,20}$ Outcome parameters were calculated using custom software blind to diagnosis or testing site. To permit averaging performance across different types of outcome parameters (e.g., accuracy and reaction time), each parameter was normalized and fit to an IQ-style scale ( mean $=100, S D=15)$ with the control group as the reference sample. Missing outcome parameter data due to a failed Mindstreams practice session was assigned a value of 2.5 standard deviations below the appropriate reference sample mean. To handle outliers, performance poorer than 2 standard deviations below the reference sample mean was replaced with a value 2 standard deviations below the mean.

The following subtests ${ }^{19}$ were included: Verbal Memory, Non-Verbal Memory, Go-NoGo, Stroop, Verbal Function, Problem Solving, Visual Spatial Imagery, Staged Information Processing, Finger Tapping, and "Catch" Game.

Normalized subsets of outcome parameters were averaged to produce six "index scores" as follows, each summarizing performance in a different cognitive domain: Memory: mean accuracies for learning and delayed recognition phases of Verbal and Non-Verbal Memory tests; Executive Function: composite scores (accuracy divided by reaction time) for Stroop test and Go-NoGo test, mean weighted accuracy for "Catch" Game; Visual-Spatial: mean accuracy for Visual Spatial Imagery test; Verbal: weighted accuracy for verbal rhyming test (part of Verbal Function test); Attention: mean reaction times for Go-NoGo and choice reaction time (Stroop, second phase) tests, mean standard deviation of reaction time for Go-NoGo test, mean reaction time for a low-load stage of Staged Information Processing test, mean accuracy for a medium-load stage of Information Processing test; Motor Skills: mean time until first move for "Catch" Game, mean inter-tap interval and standard deviation of inter-tap interval for Finger Tapping test.

A Global Cognitive Score (GCS) reflecting general cognitive status was computed as the average of the index scores. Index scores and the GCS served as dependent measures for the present study.

\section{Statistical analysis for Mindstreams data}

Because of the small sample size, Mindstreams index score and GCS performance in drug-naïve patients was compared with that of control participants using the nonparametric Mann-Whitney $U$ test. The $U$ test was also used to compare performance of untreated patients with that of patients receiving ERT or miglustat. Two-tailed statistics were used throughout, and $P<0.05$ was considered significant. All statistics were computed with SPSS statistical software (SPSS, Chicago, IL).

A Severity Score Index (SSI) is computed for each patient at baseline that reflects severity of basic disease parameters at that time plus age of onset of symptoms ${ }^{21}$; this score is never revised but remains as an index of disease involvement that may be used as a measure for comparison between patients or groups of patients. The SSI ranges from 0 to 30 points with more points added because of existence of Gaucher-specific organ involvement such as skeletal disease, pulmonary involvement, splenectomy, as well as early age of diagnosis/onset of symptoms. Generally, 0 to 10 points is consistent with mild disease; 11 to 20 points reflects moderate involvement; and 21 to 30 points is considered severe disease.

\section{RESULTS}

Table 1 shows the patient participation population in the two sets of tests. In all, 108 patients (mean age: $42.5 \pm 13.8$ years, range 19-76 years; mean years of education: $14.6 \pm 2.7$ years, range 6-22 years) participated in the traditional neuropsychological testing administered by psychologists.

Fifty-five patients (mean age: $41.6 \pm 13.0$ years, range $20-72$ years; mean years of education: $14.0 \pm 2.7$ years, range $6-22$ years) had been exposed to miglustat and were either receiving the drug as part of a clinical trial or (31 patients) had discontinued the drug at some point (e.g., due to concern about the patient with memory loss). The mean SSI for these patients was $11.1 \pm 5.6$ points (range $5-26$ points). There were 16 patients

Table 1

Patient participation population in the two sets of tests

\begin{tabular}{|c|c|c|c|c|c|}
\hline & Miglustat-treated & Enzyme-treated & Untreated & $\begin{array}{l}\text { Non-Gaucher } \\
\text { controls }\end{array}$ & Total \\
\hline $\begin{array}{l}\text { Total patients taking the psychologist } \\
\text { administered battery (homozygous } \\
\text { N370S mutation) }\end{array}$ & $55(16)$ & $31(9)$ & $22(18)$ & & $108(43)$ \\
\hline $\begin{array}{l}\text { Total patients taking the Mindstreams } \\
\text { battery (also given the psychologist } \\
\text { administered battery) }\end{array}$ & $36(33)$ & $15(13)$ & $18(11)$ & 140 & $\begin{array}{l}\text { Total patients }=69 ; \\
\text { Patients given both sets }=57 ; \\
\text { Nonpatient controls }=140 .\end{array}$ \\
\hline
\end{tabular}


Table 2

Comparison of z-scores \pm standard deviation on traditional battery

\begin{tabular}{|c|c|c|c|}
\hline Subtest & Miglustat-treated & Enzyme-treated & Untreated \\
\hline Mini-Mental State Examination (number correct of 30 points) & $28.9 \pm 1.1$ & $29.3 \pm 0.9$ & $29.3 \pm 0.8$ \\
\hline ROCFT (copy complex figure) & $-0.5 \pm 2.0$ & $-0.4 \pm 2.5$ & $8.8 \pm 1.0$ \\
\hline ROCFT (recall complex figure) & $-0.1 \pm 0.9$ & $-0.1 \pm 1.1$ & $2.0 \pm 1.0$ \\
\hline ROCFT (\% recalled) & $-0.1 \pm 1.0$ & $-0.1 \pm 1.0$ & $1.0 \pm 1.0$ \\
\hline RAVLT4 & $-0.8 \pm 1.4$ & $-0.4 \pm 1.3$ & $-0.4 \pm 1.1$ \\
\hline RAVLT5 & $-0.8 \pm 1.4$ & $-0.3 \pm 1.2$ & $-0.3 \pm 0.9$ \\
\hline RAVLT7* $^{*}$ & $-0.56 \pm 0.94$ & $0.07 \pm 0.7$ & $-0.17 \pm 0.85$ \\
\hline RAVLT8* & $-0.61 \pm 0.82$ & $0.08 \pm 0.84$ & $-0.43 \pm 0.85$ \\
\hline FAS $^{*}$ (number responses correct) & $31.54 \pm 11.3$ & $38.52 \pm 10.5$ & $36.20 \pm 10.3$ \\
\hline Animals* (number responses correct) & $19.54 \pm 5.65$ & $22.55 \pm 5.72$ & $21.84 \pm 4.83$ \\
\hline Trail Making Test A (connecting dots with numbers) & $-1.1 \pm 1.6$ & $-0.4 \pm 1.4$ & $-0.5 \pm 1.2$ \\
\hline Trail Making Test $\mathrm{B}^{*}$ (connecting dots of letters alternating with numbers) & $-1.08 \pm 1.88$ & $0.06 \pm 1.38$ & $-0.25 \pm 1.62$ \\
\hline Tower of Hanoi (time in seconds) & $145.8 \pm 349$ & $85.6 \pm 71$ & $81.8 \pm 68$ \\
\hline Tower of Hanoi (number of steps) & $9.3 \pm 3.5$ & $11.2 \pm 8.2$ & $8.1 \pm 2.0$ \\
\hline Wisconsin Card Sorting (category recognition) & $-1.2 \pm 1.8$ & $-0.9 \pm 1.7$ & $-0.7 \pm 1.4$ \\
\hline Wisconsin Card Sorting (perseverance) & $-0.9 \pm 1.8$ & $-0.8 \pm 1.8$ & $-0.8 \pm 1.4$ \\
\hline Wisconsin Card Sorting (failure to maintain set) & $-0.2 \pm 1.1$ & $-0.3 \pm 1.0$ & $-1.3 \pm 0.8$ \\
\hline Wisconsin Card Sorting (\% of conceptual level responses) & $-2.0 \pm 1.6$ & $-1.8 \pm 1.5$ & $-1.9 \pm 1.3$ \\
\hline
\end{tabular}

Other than Mini-Mental, FAS, Animals, and Tower of Hanoi where units are noted in parentheses.

${ }^{\star}$ Statistically significant differences.

(29.1\%) who were homozygous for the N370S [1226G] mutation.

Thirty-one patients (mean age: $42.5 \pm 15.1$ year, range $20-71$ years; mean years of education: $14.9 \pm 2.8$ years, range 7-19 years) were receiving ERT. The mean SSI for these patients was $11.0 \pm 5.8$ points (range 5-24 points). There were 9 patients $(29.0 \%)$ who were homozygous for the N370S [1226G] mutation (see Table 1).

Twenty-two patients (mean age: $44.9 \pm 14.5$ years, range 19-76; mean years of education: $15.6 \pm 2.1$ year, range 12-19 years) were drug-naïve (i.e., never treated with either drug; "untreated"). The mean SSI for these patients was $6.6 \pm 3.8$ points (range $2-14$ points). There were 18 patients $(81.8 \%)$ who were homozygous for the N370S [1226G] mutation (see Table 1).

There was no difference between groups with regard to age or years of education.

There was no difference between the miglustat and ERT groups with regard to SSI or prevalence of homozygosity for the N370S mutation. However, comparison of either group with the untreated group was statistically significant for SSI $(U=221.0, P=0.001$; and $U=144.5, P=0.003$, respectively). Similarly, there was borderline statistical significance between miglustat and ERT groups to the untreated group with regard to prevalence of homozygosity for the N370S mutation ( $P=0.053$ and $P=0.049$, respectively).
Some tests were aborted by patients due to time constraints; the greatest number of unfinished subtests was 12/55 patients receiving miglustat who aborted the subtests RAVLT7 and RAVLT8.

In the psychologist-administered battery, mean scores in all subtests and for all groups were consistently more than one z-score below normal (Table 2). Between-group comparisons were nonsignificant for $13 / 18$ subtests. There were only 5 subtests with statistically significant differences between groups by ANOVA when using $-2.0 \mathrm{z}$-scores as cut-off points and taking ERT and untreated groups together versus miglustat-treated patients (Table 2). For verbal learning and memory, patients exposed to miglustat performed significantly poorer than patients in the other groups (RAVLT7, $P=0.013$ and RAVLT8, $P=0.005$ ) For verbal fluency, patients exposed to miglustat performed significantly poorer than other groups (FAS, $P=0.018$ and Animals, $P=0.045$ ). For visuospatial functioning in the Trail Making Test B, patients exposed to miglustat performed less well than the other groups (TMTB, $P$ $=0.011)$. Despite the reduced performance in the miglustat group in these subtests, Pearson chi-square test revealed no correlation between performance and duration of miglustat therapy.

Sixty nine patients (mean age: $47.8 \pm 13.4$ years; mean years of education: $14.5 \pm 2.4$ years) participated in Mindstreams computerized testing. Thirty-six patients had been exposed to 
miglustat and were either receiving the drug (25 patients, 69\%) at the time of testing or had discontinued the drug (11 patients; $31 \%$ ) due to suspension of the clinical trial. Thirty three of these miglustat patients also had participated in the psychologist-administered testing (see Table 1). Fifteen patients were receiving ERT, 13 of whom also participated in the psychologist-administered battery. Eighteen patients were drug-naïve, 11 of these patients also participated in the psychologist-administered battery. The control group consisted of 140 individuals diagnosed as cognitively healthy in controlled research studies at nine clinical sites. Control participants were comparable to patients with Gaucher disease in age $(U=4698.5, P=$ $0.749)$, years of education $(U=4768.5, P=0.878)$, and gender $\left(\chi^{2}[1, N=209]=0.337, P=0.561\right)$.

Mindstreams performance in drug-naïve patients was not significantly different from normal controls $(P>0.05)$ across all cognitive domains. Patients receiving ERT performed similarly to the drug-naïve group $(P>0.05)$ in all domains. Patients in the miglustat group performed similarly to the drugnaïve group $(P>0.05)$ in all domains but visuospatial function, for which performance was significantly better $(P<$ 0.05). Although not statistically significant, means in the ERT group were consistently lower than in the drug-naïve group, whereas means in the miglustat group were consistently higher than baseline (Fig. 1).

\section{DISCUSSION}

Gaucher disease has traditionally been subdivided into "types" based on the presence or apparent absence of neurological manifestations. As experience with Gaucher disease has extended worldwide, it is increasingly clear that, within each classical type, there is a spectrum of clinical signs and symptoms. Even as regards neuronopathic disease, genotype analysis is frequently useful yet insufficient for reliably predicting

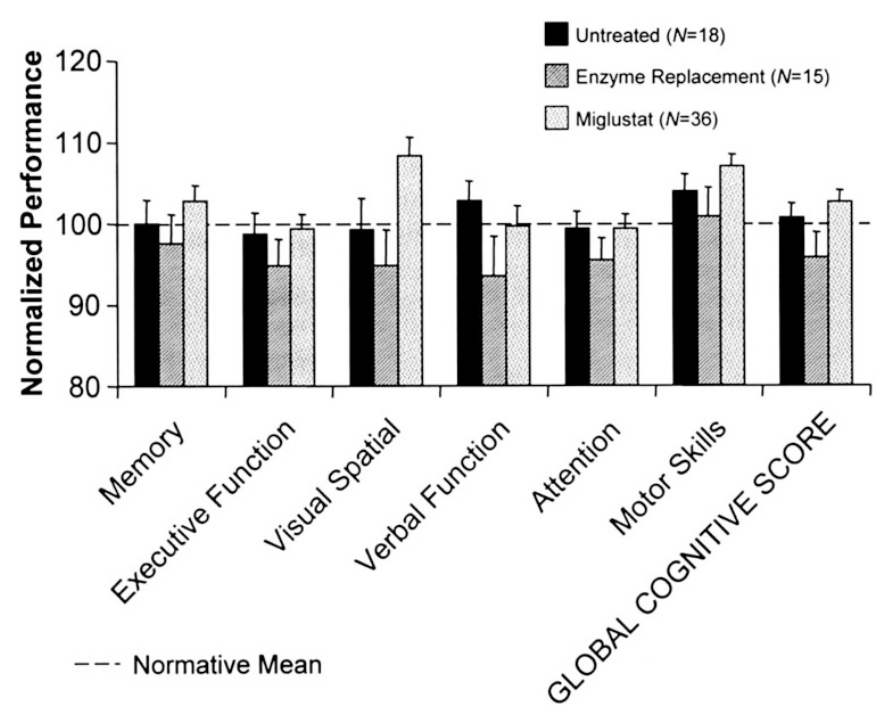

Fig. 1. Performance on Mindstreams index scores and Global Cognitive Score by patients with type I Gaucher disease. phenotypic expression, course of disease, and prognosis. There are patients with homozygosity or compound heterozygosity for mutant alleles that are typically associated with type 2 or type 3 Gaucher disease who are reported free of apparent neurological dysfunction. However, these patients must be closely monitored for late onset of neurological dysfunction as illustrated by the case history of a child of Kurdish ethnicity (homozygous for the L444P [1448C] mutation) who was the first child to receive ERT in Israel. There was no overt evidence of neurological involvement at age 2 years. After 4 years of ERT, at age 6 years, neuropsychological evaluation revealed low-normal intelligence and slow motor development. At age 11 years, horizontal gaze palsy was overt and PET brain scan revealed slightly decreased perfusion in the right parietal lobe.

The presence of even one N370S [1226G] mutation is commonly thought to be invariably predictive of nonneuronopathic (type 1) Gaucher disease. However, there are now reports of phenomena such as tremor and other extrapyramidal movement abnormalities, peripheral neuropathy, and age-inappropriate cognitive dysfunction that may possibly be part of the natural history even in patients believed to be free of neurological risk. Adult patients were described with a Parkinsonian syndrome of early-onset but progressive course with refractoriness to standard (levodopa) therapy.22-24 In a neuropathological study of brains of patients with Gaucher disease, hippocampal areas CA2-4 and layer $4 \mathrm{~b}$ of the calcarine cortex showed astrogliosis in 7 subjects with type 1 Gaucher disease 4 of whom had Parkinson's disease with dementia. ${ }^{25}$ In unpublished studies by our group (Levy-Lahad and Domb et al., 2004) that asked the question whether carriers of Gaucher disease enjoy a selective advantage of increased intelligence, we administered the Wechsler Intelligence Scale and Raven Matrices to appropriately age- and sex-matched carrier and normal siblings of adults and children with Gaucher disease. Both groups had high intelligence scores, but the carriers surprisingly had significant visuospatial deficits. These findings were not dissimilar to those reported among carriers of metachromatic leukodystrophy and Krabbe disease. ${ }^{26,27}$ Thus, some patients may exhibit signs or symptoms that blur the demarcation lines between clinical types and, more accurately, are part of a range of possible phenotypes from nonneurological to life-threatening neurological involvement. This observation may be particularly important for the evaluation of new, small molecule therapies for Gaucher disease such as miglustat, which, unlike imiglucerase, are permeable through the bloodbrain barrier and may have potential for neurotoxicity as well as therapeutic potential.

Our study of cognitive function in patients with type $1 \mathrm{Gau}-$ cher disease with standardized neuropsychological tests that emphasize visuospatial functioning unexpectedly found low scores in all groups and on all subtests. Moreover, patients treated with miglustat had significantly lower scores on a few of these psychologist-administered neuropsychological battery than either treatment-naïve patients or patients treated with ERT. On the other hand, these differences were not apparent with the computerized test battery where the patients' results 
were within normal limits and comparable to well-matched healthy controls. It is therefore plausible that the specificity of the traditional battery as administered resulted in a less representative picture of overall cognitive function by virtue of highlighting the dysfunctional visuospatial performance. The second testing battery, using the computerized system, was chosen for its brevity and objectivity relative to the individualized psychologist-administered battery. The computerized battery was also selected because of the availability of multiple test languages and language/culture appropriate normative data. The computerized system by virtue of matched control groups provided a more rigorous evaluation of performance, while again highlighting visuospatial indices.

Our neuropsychological test results are consistent with the conventional wisdom and extant experience that development of cognitive dysfunction is not a feature of the natural history of type 1 Gaucher disease. Any dysfunction suggested by the current study is apparently subtle and of doubtful clinical relevance given that cognitive status did not interfere with patients' daily function; among the patients were physicists and architects, professions relying upon visuospatial skills. Our results also suggest that treatment of Gaucher disease with miglustat does not lead to cognitive impairment. This conclusion, based on results from the traditional neuropsychological battery, allowed reinstatement of the trial at our clinic and eventual licensing of the investigational drug for marketing.

It is important to note that patients who had been exposed to miglustat did perform significantly poorer than the other groups on measures of verbal learning and memory, verbal fluency, and complex symbol alteration sequencing on psychologist administered tests. But these patients did not perform significantly worse in these skills in the Mindstreams battery. Although the data from both psychologist-administered and computer-assessed tests suggest no significant adverse effect of miglustat over other treatments on executive function and visuomotor/visuospatial abilities, data from the psychologist administered tests may be suggestive of greater risk for verbal and memory difficulties. Because the precipitating event for our Ethics Committee review of miglustat did not involve visuomotor/visuospatial abilities per se, but rather the complaints of a specific patient (see earlier), the verbal/memory results from the psychologist administered batteries may have relevance only to the safety question. Nonetheless, in that the Mindstreams battery, albeit in a more cursory way, screens these same functions, the difference in functional domains identified in the two parts of the study is not qualitatively different.

The rationale for combining the nonmiglustat groups for comparison with the miglustat group in the psychologist-administered battery was a consequence of the impetus of the evaluation by the Ethics Committee, i.e., to insure that the cognitive dysfunction in the index case was not pervasive among patients exposed to the heretofore untried modality of substrate reduction. This statistical manipulation therefore was not because of any implicit comparability with regard to disease severity between ERT-treated and untreated groups but rather to bring to light any cognitive change that was emergent due to miglustat exposure.

A further complication in interpreting the results of the psychologist-administered battery relative to the computer-assessed battery is the high percentage of technical failure in some of the psychologist-administered subtests, e.g., $22 \%$ of patients on miglustat aborted RAVLT 7 and RAVLT8. This did not occur in the computer-assessed battery. Hence, although one may query whether these subtests were too difficult and/or that the patients had insufficient time, some statistically significant differences between the two sets may reflect technical difficulties inherent to the mode of administration. As suggested, this finding underscores the fact that apparent statistical significance may not have a correlate in clinical reality.

Thus, in addition to the need for longitudinal studies, larger sample sizes are required before definitive conclusions can be drawn. Similarly, any ameliorative effect of miglustat upon cognitive function, as was hinted at in the computerized testing, must also await a larger sample size. The computerized test battery used in the current study is well-suited for longitudinal testing and for long-term surveillance of miglustat treatment as required by the regulatory agencies. Such studies of miglustat are currently underway in our clinic.

Comparison of cognitive testing results between Gaucher disease and other lysosomal disorders is problematic due to lack of a uniform, standardized test battery. A recent neuropsychological assessment of patients with late onset $\mathrm{GM}_{2}$ gangliosidosis showed no deterioration in visuospatial functioning, whereas memory and executive functioning was more impaired. ${ }^{28}$ Although this study used a psychologist-administered battery largely comparable to that of the present study, differences in choice of tests, test-administration, and diseasespecific physical disabilities make comparison with our results difficult. Indeed, it is also for the purpose of comparing results across disease groups that we recommend adoption of standardized computerized cognitive testing as a screening test for long-term surveillance. In addition to better comparability across studies and other advantages outlined here, computerized cognitive testing with Mindstreams is also suitable for children and teenagers with incipient neurological involvement such as in type 3 Gaucher disease and juvenile forms of other lysosomal storage disorders. To date, standard tools to assess progression of cognitive dysfunction and/or response to therapy in these groups have not been applied; albeit for in-depth evaluation (of the brain-behavior relationship) and developing rehabilitative recommendations, individualized traditional neuropsychologist-administered battery should be preferred.

Given that the neurological changes in type 1 Gaucher disease and/or due to exposure to treatment regimens reflected in the current study are subtle and clinically inconsequential relative to the more overt changes associated with type 2 and type 3 Gaucher disease, it seems appropriate and more clinically useful to continue to subdivide Gaucher disease into the classic types rather than adopt a new continuum of signs and symptoms model. Although evidence supports a continuum of clinical manifestations spanning the gap between types 2 and 3 and 
between types 1 and 3 , it is still a critical distinction that the classical neuronopathic forms involve evident and reproducible patterns of neurological and cognitive decline. Evaluation of performance in all these patients, however, could be facilitated and would benefit from a unified and standardized testing protocol.

\section{ACKNOWLEDGMENTS}

The funding for this study was derived in part by grants from the Oxford GlycoScience Company, Oxford UK and the Teva Pharmaceutical Industries Ltd, Netanya, Israel. This study was accepted for presentation at the first meeting of the WORLD Conference in Minneapolis, June 2004. The authors would like to acknowledge the professionalism and dedication of our biostatistician Tali Bdolah-Abram of the Hebrew University, Jerusalem. The authors wish to dedicate this article to the memory of Dr. Philip Rosenberg, the neurologist involved in these studies in the early stages, who passed away suddenly in August, 2003.

\section{References}

1. Barton NW, Brady RO, Dambrosia JM, Di Bisceglie AM, Doppelt SH, Hill SC, Mankin $\mathrm{HJ}$ et al, and collaborators. Replacement therapy for inherited enzyme deficiency: macrophage-targeted glucocerebrosidase for Gaucher's disease. N Engl J Med 1991;324:1464-1470.

2. Weinreb NJ, Charrow J, Andersson HC, Kaplan P, Kolodny EH, Mistry P et al. Effectiveness of enzyme replacement therapy in 1028 patients with type 1 Gaucher disease after 2 to 5 years of treatment: a report from the Gaucher Registry. Am J Med 2002;113:112-119.

3. Radin NS. Treatment of Gaucher disease with an enzyme inhibitor. Glycoconj J 1996;13:153-157.

4. Cox T, Lachmann R, Hollak C, Aerts J, van Weely S, Hrebicek M et al. Substrate reduction using $N$-butyldeoxynojirimycin (OGT 918): Clinical trial of a novel oral therapy for Gaucher's disease. Lancet 2000;355:1481-1485.

5. Heitner R, Elstein D, Aerts J, van Weely S, Zimran A. Low-dose N-butyldeoxynojirimycin (OGT 918) for type I Gaucher disease. Blood Cells Mol Dis 2002;28:127-133.

6. Elstein D, Dwek A, Attias D, Hadas-Halpern I, Zevin S, Altarescu G et al. OGT 918 as an oral maintenance monotherapy or in combination with enzyme replacement therapy (ERT) in adult patients with Gaucher disease. 5th EWGGD, Prague May 2002

7. Cox TM, Aerts JM, Andria G, Beck M, Belmatoug N, Bembi B et al. Advisory Council to the European Working Group on Gaucher Disease. The role of the iminosugar N-butyldeoxynojirimycin (miglustat) in the management of type I (non-neuronopathic) Gaucher disease: a position statement. J Inherit Metab Dis 2003;26:513-526.

8. Pastores GM, Barnett NL. Substrate reduction therapy: miglustat as a remedy for symptomatic patients with Gaucher disease type 1. Expert Opin Investig Drugs 2003; 12:273-281.

9. European Public Assessment Report (EPAR). Zavesca [miglustat]. Committee for Proprietary Medicinal Products. CPMP/3795/02;2002 Available at: http://www.e-
mea.eu.int/humandocs/Humans/EPAR/zavesca/zavesca.htm. Accessed November $11,2004$.

10. Package Insert, Zeneca [miglustat], Capsules, 100mg, 31 July 2003. Available at: http://www.fda.gov/cder/foi/nda/2003/21-348_Zavesca_Prntlbl.pdf. Accessed November 11, 2004.

11. Ward LC. Prediction of verbal, performance, and full scale IQs from seven subtests of the WAIS-R. J Clin Psychol 1990;46:436-440.

12. Folstein MF, Folstein SE, McHugh PR. "Mini-mental state": A practical method for grading the cognitive state of patients for the clinician. J Psychiatr Res 1975;12:189198.

13. Spreen O, Strauss E. A compendium of neuropsychological tests (2nd ed.) Oxford University Press, New York; 1998.

14. Bennett-Levy J. Determinants of performance on the Rey-Osterrieth Complex Figure Test: an analysis, and a new technique for single-case assessment. Br J Clin Psychol 1984;23(pt 2):109-119.

15. Gordon NG. Diagnostic efficiency of the Trail Making Test as a function of cut-off score, diagnosis, and age. Percept Mot Skills 1978;47:191-195.

16. Mitrushina M, Satz P, Chervinsky A, D’Elia L. Performance of four age groups of normal elderly on the Rey Auditory-Verbal Learning Test. J Clin Psychol 1991;47: 351-357.

17. Parks RW, Cardoso J. Parallel distributed processing and executive functioning: Tower of Hanoi neural networkmodel in healthy controls and left frontal lobe patients. Int J Neurosci 1997;89:217-240.

18. Anderson SW, Damasio H, Jones RD, Tranel D. Wisconsin Card Sorting Test performance as a measure of frontal lobe damage. J Clin Exp Neuropsychol 1991;13:909_ 922.

19. Dwolatzky T, Whitehead V, Doniger GM, Simon ES, Schweiger A, Jaffe D, Chertkow $\mathrm{H}$. Validity of a novel computerized cognitive battery for mild cognitive impairment. BMC Geriatr 2003;3:4.

20. Schweiger A, Doniger GM.,. Dwolatzky T, Jaffe D, Simon ES. Reliability of a novel computerized neuropsychological battery for mild cognitive impairment. Acta Neuropsychologica 2003;1:407-413.

21. Zimran A, Sorge J, Gross E. Prediction of severity of Gaucher's disease by identification of mutations at DNA level. Lancet 1989;2:349-353.

22. Neudorfer O, Giladi N, Elstein D, Abrahamov A, Turezkite T, Aghai E et al. Occurrence of Parkinson's syndrome in type I Gaucher disease. Quart J Med 1996;89:691694.

23. Perez-Calvo J, Bernal M, Giraldo P, Torralba MA, Civeira F, Giralt M et al. Comorbidity in Gaucher's disease results of a nationwide enquiry in Spain. Eur J Med Res 2000;5:231-235.

24. Varkonyi J, Rosenbaum H, Baumann N, MacKenzie JJ, Simon Z, Aharon-Peretz J, Walker JM, Tayebi N, Sidransky E. Gaucher disease associated with parkinsonism: four further case reports. Am J Med Genet A. 2003;16:348-351.

25. Wong K, Sidransky E, Verma A, Mixon T, Sandberg GD, Wakefield LK, Morrison A, Lwin A, Colegial C, Allman JM, Schiffmann R. Neuropathology provides clues to the pathophysiology of Gaucher disease. Mol Genet Metab. 2004;82:192-207.

26. Christomanou H, Martinius J, Jaffe S, Betke K, Forster C. Biochemical, psychometric, and neuropsychological studies in heterozygotes for various lipidoses. Preliminary results. Hum Genet. 1980;55:103-110.

27. Christomanou H, Jaffe S, Martinius J, Cap C, Betke K. Biochemical, genetic, psychometric, and neuropsychological studies in heterozygotes of a family with globoid cell leucodystrophy (Krabbe's disease). Hum Genet. 1981;58:179-83.

28. Zaroff CM, Neudorfer O, Morrison C, Pastores GM, Rubin H, Kolodny EH. Neuropsychological assessment of patients with late onset GM2 gangliosidosis. Neurology. 2004;62:2283-2286. 